Original Article - Clinical Science

Funding Information: Angior Family Foundation Fund, (Grant / Award Number: ) Australia National Health and Medical Research Council , (Grant / Award Number: ' ','GNT1104700') Australia National Health and Medical Research Council Senior Research Fellowship, (Grant / Award Number:

'1138585') Keratoconus Australia Fund, (Grant / Award Number: ) Lions Eye Foundation Fellowship, (Grant / Award Number: )

\title{
Economic impact of keratoconus using a health expenditure questionnaire: a patient perspective
}

\author{
Elsie Chan FRANZCO, 1,2,3* Paul N. Baird PhD, ${ }^{1,2 *}$ Sara Vogrin MBiostat, ${ }^{2,4}$ Vijaya \\ Sundararajan MD, ${ }^{4,5}$ Mark D. Daniell FRANZCO ${ }^{1,2,3 \#}$ and Srujana Sahebjada PhD ${ }^{1,2 \#}$
}

*Elsie Chan and Paul Baird contributed equally as $1^{\text {st }}$ authors to this paper

\# Mark Daniell and Srujana Sahebjada contributed equally as senior authors

${ }^{1}$ Centre for Eye Research Australia, Melbourne, Australia

2 Ophthalmology, Department of Surgery, The University of Melbourne, Melbourne, Australia,

${ }^{3}$ Royal Victorian Eye and Ear Hospital, Melbourne Australia,

4 St. Vincent's Hospital, Melbourne Australia

5 Department of Public Health, La Trobe University, Melbourne Australia.

Correspondence: Dr Srujana Sahebjada, Centre for Eye Research Australia, Level 7, Royal Victorian Eye and Ear Hospital, 32 Gisborne Street, East Melbourne, VIC, 3002 Australia

Email: Srujana.sahebjada@unimelb.edu.au

Short running title: Economic burden of keratoconus

Received 17 J une 2019; accepted 14 December 2019

This is the author manuscript accepted for publication and has undergone full peer review but has not been through the copyediting, typesetting, pagination and proofreading process, which may lead to differences between this version and the Version of Record. Please cite this article as doi: 10.1111/ceo.13704

This article is protected by copyright. All rights reserved. 
Funding sources / Financial disclosure: This study was supported by the Australian National Health and Medical Research Council (NHMRC) project grant GNT1104700 and Senior Research Fellowship (1138585 to PN Baird), the Angior Family Foundation, Keratoconus Australia and a Lions Eye Foundation Fellowship (SS). The Centre for Eye Research Australia (CERA) receives Operational Infrastructure Support from the Victorian Government.

Conflict of interest: None

\section{ABSTRACT}

I mportance: This is the first study to estimate the lifetime costs associated with keratoconus based on a questionnaire completed by patients and highlights the significant economic burden of the disease. As keratoconus affects individuals from a young age, the study highlights keratoconus as a public health concern.

Background: Keratoconus is a disorder characterised by corneal steepening and thinning, leading to reduced visual acuity. To date, there have been no studies evaluating the economic costs of keratoconus from a patient's perspective.

Design: A randomised cross-sectional study undertaken in Australia where keratoconus subjects were recruited from public and private ophthalmology and optometry clinics.

Participants: A total of 100 participants completed the questionnaire: median age was 31 years and $57 \%$ were males.

Methods: A keratoconus health expenditure questionnaire was designed to assess direct and indirect expenditures for each individual.

Main Outcome Measures: Total direct and indirect costs associated with the condition were calculated along with the estimated lifetime per capita costs.

Results: The total cost related to direct and indirect care was estimated to be AUD 3365. By applying our cost data to keratoconus prevalence data for the Australian 
population, the total cost is estimated to be approximately AUD 44.7 million per year in Australia.

Conclusions and Relevance: Our results show that the costs associated with the diagnosis and management of keratoconus represent a significant cost to patients. An understanding of this is important not only to individuals and their families, but also health care providers, health insurers and the wider health system.

Keywords: Keratoconus, Cornea, Corneal graft, Corneal transplantation 


\section{I NTRODUCTION}

Keratoconus $(\mathrm{KC})$ is a condition that is characterised by bilateral, progressive corneal thinning that results in an abnormally steep cornea, irregular astigmatism and decreased vision ${ }^{1}$.

In the early stages of $\mathrm{KC}$, vision is correctable with spectacles, but with progression of the condition, rigid contact lenses are usually required. While these aids correct the refractive error associated with $\mathrm{KC}$, they do not treat the underlying cause of the disease, and therefore do not affect disease progression. Corneal cross-linking (CXL) has been available in Australia for a decade and has been shown to halt the progression of $\mathrm{KC}$ in most treated patients ${ }^{2-7}$. The procedure involves application of topical riboflavin drops and ultraviolet- $A$ irradiation to the cornea. Currently, $C X L$ is being used in most parts of the world for the management of progressive KC. However, in many countries, the cost of this treatment is not covered by the government or health insurers.

In advanced cases of KC where the cornea becomes extremely thin and scarred, corneal transplantation surgery may be necessary to restore vision. $\mathrm{KC}$ is the most common indication for corneal transplantation in Australia, accounting for around 30\% of corneal grafts across the country, and almost $50 \%$ of corneal transplants performed in the state of Victoria in Australia ${ }^{8}$. A recent study from the Australian Corneal Graft Registry estimated that a KC patient who undergoes corneal transplantation will typically require up to five transplants over their lifetime9.

In Australia, the cost of spectacles and contact lens prescriptions are only partially covered by limited State-based subsidy schemes and private health insurers. Furthermore, CXL was only added to Australia's Medicare Benefits Schedule on $1^{\text {st }}$ 
May, 2018. Prior to this date, patients had limited access to the treatment without payment through only selected hospitals in Australia, with most patients paying for the treatment without any government rebate. Thus, patients with KC have been required to pay significant out-of-pocket costs for their treatment and management.

Rebenitsch et al. estimated the expected incremental lifetime cost of treatment of KC in USA as \$25 $168{ }^{10}$. However, this model relied on published sources of data, consultation with physicians and the authors' own estimations. For the development of cost-effectiveness or cost-utility analyses, the true cost of $\mathrm{KC}$ incurred by the individual and by the community must be known. Thus, the aim of this study was to evaluate the economic cost associated with $\mathrm{KC}$ using a questionnaire to calculate the annual costs and project economic burden associated with the disease.

\section{METHODS}

\subsection{Participants}

A cross-sectional study of KC patients was undertaken in Melbourne, Australia from October 2015- May 2016. Patients were recruited from private rooms, public clinics at the Royal Victorian Eye and Ear Hospital and optometry clinics in Melbourne. A patient information sheet and consent form were provided to all individuals participating in the study. The study protocol was approved by the Royal Victorian Eye and Ear Hospital Human Research and Ethics Committee (Project 10/954H). This study followed the tenets of the Declaration of Helsinki and all privacy requirements were met.

All KC patients presenting to the clinics were invited to participate in the study which involved completing a questionnaire and undergoing ocular examination and imaging including using the Pentacam (Oculus $\mathrm{GmbH}$, Wetzlar, Germany). Inclusion and 
exclusion criteria for $\mathrm{KC}$ and details of the clinical examination for this study have been described elsewhere ${ }^{11-13}$. In brief, $\mathrm{KC}$ was diagnosed based on the presence of one or more of the following: an irregular cornea on manual keratometry and/or Pentacam imaging; scissoring of the retinoscopic reflex; demonstration of at least one biomicroscopic sign, including Vogt's striae, Fleischer ring, or corneal thinning consistent with $\mathrm{KC}$.

\subsection{Keratoconus health expenditure questionnaire}

The questionnaire was designed to assess expenditure on $\mathrm{KC}$ by the participants over the preceding 12 months. The questionnaire was set up as an on online survey using REDCap (Research Electronic Data Capture). REDCap is a secure, web-based application designed to support data capture for research studies, providing 1) an intuitive interface for validated data entry; 2) audit trails for tracking data manipulation and export procedures; 3) automated export procedures for seamless data downloads to common statistical packages; and 4) procedures for importing data from external sources. All study data were collected, managed and stored using REDCap hosted at the Centre for Eye Research Australia ${ }^{14}$.

Costs not claimable through the Australian Government's Medicare system or private health insurers, referred to as 'out-of-pocket' costs, were recorded for visual aids (spectacles, contact lenses and contact lens related products). Any costs relating to private health insurance (including premiums) were documented. Participants were also asked about their satisfaction with the optical benefits provided by their private health insurer and any additional expenses they felt should be covered which were not currently included.

Direct costs related to the treatment of $\mathrm{KC}$ were calculated by asking the participants how many times during the past year they had received care from an optometrist, 
ophthalmologist or hospital; the reason for the visit and if there were any associated out-of-pocket costs (such as 'gap' fees or transportation costs). Costs related to any surgical treatment (e.g. CXL, cornea transplantation), and the purchase of any medications, products or equipment were also recorded. Additional costs as the result of visits to other health practitioners for purposes related to their diagnosis of KC (e.g. general practitioners and psychologists) were also included. The questionnaire then covered the costs relating to informal care and support that participants faced in managing their KC. Details on the type of care, provider/helper and the cost to participant/s or the helper were obtained. (E.g. Transport - Husband - \$30 (selfpetrol)). Data was also collected on the purchase of any prescription medicines (e.g eye drops); products or devices (e.g. low vision devices, including modified television or computer screens) and the related costs to deal with the condition in the preceding year. Finally, information was collected on each individual's employment status, household income and, in the preceding year, an estimation of the monetary value for their time away from employment, and other inconveniences caused by their diagnosis (Questionnaire included in Appendix).

\subsection{Statistical Analysis}

All analyses were performed using Stata Statistical Software (Release 13, 2013; Statacorp LP, College Station, TX). Descriptive statistics are presented as medians and interquartile ranges given the skewed distribution of the data. The estimated lifetime per capita cost was calculated as a cumulative sum of median yearly costs for each year following $\mathrm{KC}$ diagnosis. It did not take into account changes in prices over time. The cost estimate of KC Australia-wide was based on a disease prevalence of 54.5 per 100000 population ${ }^{15}$ and a population size of 24 million $^{16}$.

\section{RESULTS}


A total of 104 subjects were approached randomly of which 100 participants completed the $\mathrm{KC}$ health expenditure questionnaire (response rate $96 \%$ ). The median age of the participants was 31 years (IQR 23,44) and 57\% were males. The cost of spectacles was reported by 74 participants. On average, participants purchased one pair of spectacles in the preceding year, and their median out-of-pocket cost was AUD 237.5 (Table 1). A total of 96/100 participants provided responses on the timing of their most recent spectacle change. This occurred less than 6 months earlier for 25 participants (26\%), 6 to 12 months earlier for 34 participants (36\%), 12 to 24 months earlier for 6 participants ( $6 \%$ ), and more than 24 months earlier for 31 participants (32\%).

The majority of participants $n=62(61 \%)$ wore contact lenses on a daily basis; $7(7 \%)$ used them several times a week, 8 (8\%) on an occasional basis, and $23(23 \%)$ never used contact lenses. Of those who wore contact lenses, all but one reported the associated costs. The median out of pocket costs for contact lenses was AUD 600 and for contact lens-related care AUD 180 per annum, respectively (Table 1).

Of the 100 participants, 55 (55\%) had private health insurance and paid a median insurance premium of AUD 1100 per annum. The majority (78\%) were not satisfied with their optical cover, with participants naming contact lens solutions (46\%), contact lens fitting fees (53\%), surgical fees (59\%) and eye drops (36\%), as the most relevant items that were not completely covered by insurers (Table 1 ).

Table 1. Results of the health expenditure questionnaire. The total number of responses for each question was 100 unless otherwise stated. Costs reported are in Australian dollars (AUD). IQR, interquartile range; $\mathrm{KC}$, keratoconus.

\section{Median (IQR)}




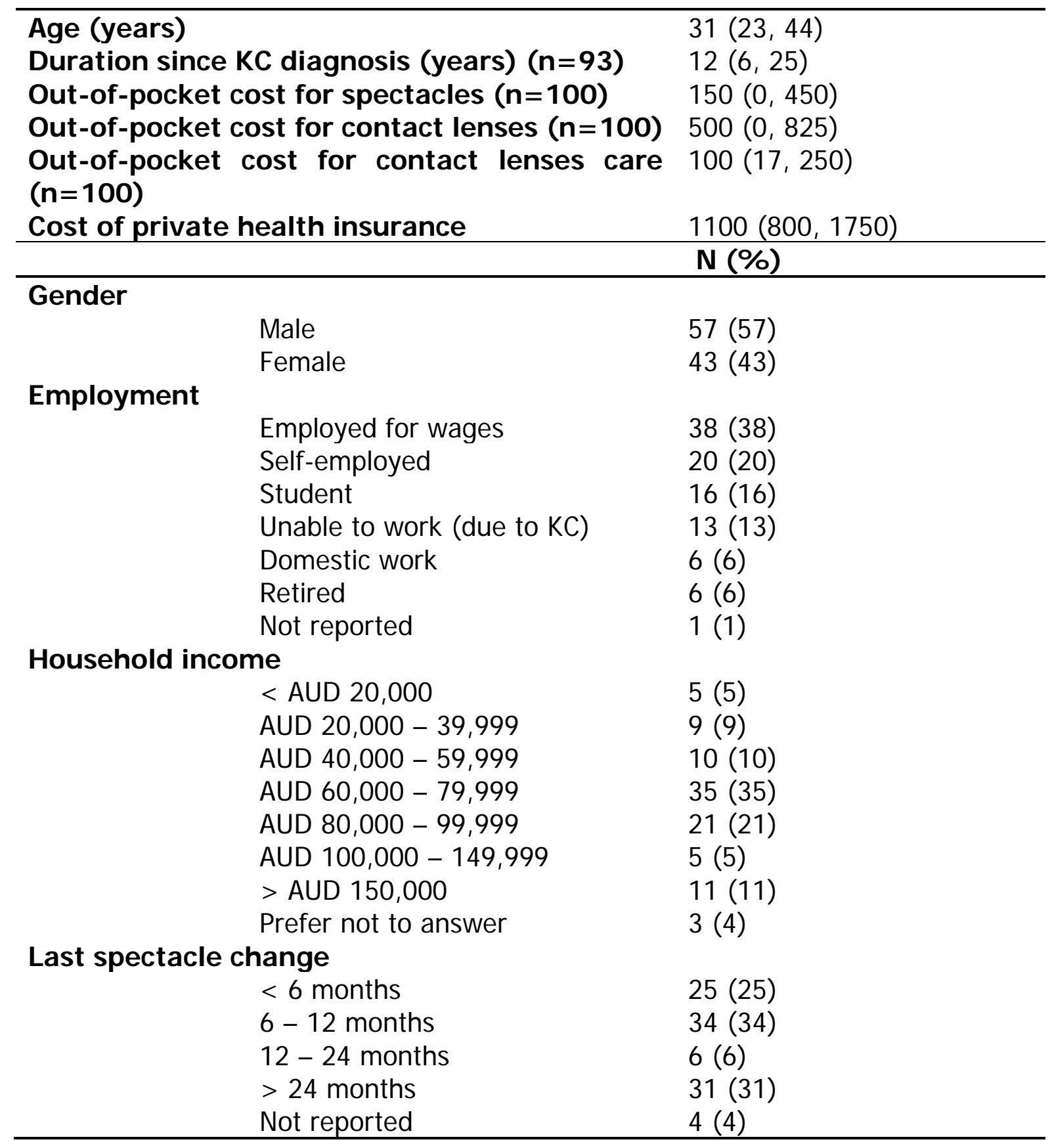

The number of participants who sought care from optometrists, ophthalmologists and/or a hospital in the past year are shown in Table 2. Additionally, the number and main purpose of each visit, as well as transportation and out-of-pocket expenses for the visits are also shown. 
Table 2: Services (optometrists, ophthalmologists and hospital clinics) and out-ofpocket costs. The total number of responses for each question was 100. Costs reported are in Australian dollars (AUD). IQR, interquartile range; KC, keratoconus.

\begin{tabular}{cl}
\hline & $\begin{array}{l}\text { N (\%) } \\
\text { (IQR) }\end{array}$ \\
\hline $\begin{array}{c}\text { Optometrist } \\
\text { Visit to optometrist }\end{array}$ & Median \\
Yes & $83(83)$ \\
No & $17(17)$ \\
Number of visits & $19(23)$ \\
1 & $22(26)$ \\
2 & $41(50)$ \\
$\geq 3$ & $1(1)$ \\
Not reported & \\
Purpose of visit & $53(64)$ \\
Contact lens fitting & $37(45)$ \\
Follow up appointment & $22(27)$ \\
Prescription check for spectacles & $50(26,100)$ \\
Cost of transportation, AUD & $70(15,200)$ \\
Out of pocket expenses, AUD & \\
Ophthalmologist & \\
Visit to ophthalmologist & $45(45)$ \\
Yes & $54(54)$ \\
No & $1(1)$ \\
Not reported & $9(20)$ \\
Number of visits & $7(16)$ \\
1 & $28(62)$ \\
2 & $1(2)$ \\
$\geq 3$ & $26(58)$ \\
Not reported & $13(29)$ \\
Purpose of visit & $12(27)$ \\
Surgery & $100(50,200)$ \\
Follow up appointment & $905(222.5,3612.5)$ \\
Routine check up & \\
Cost of transportation, AUD & \\
Out of pocket expenses, AUD & \\
&
\end{tabular}




\section{Hospital}

Eye Hospital visits

Yes $52(52)$

No 48 (48)

Number of visits

1

20 (39)

2

8 (15)

$\geq 3$

$22(42)$

Not reported 2 (4)

Purpose of visit

Routine check up 23 (44)

Follow up appointment 20 (38)

Surgery 19 (37)

Cost of transportation, AUD $\quad 60(27,150)$

Out of pocket expenses, AUD $\quad 70(0,210)$

Fourteen participants (14\%) received care for their KC from other health practitioners, with $64 \%$ also visiting their general practitioner; 2 (14\%) participants consulted a psychologist and 2 (14\%) utilised social support services. Over half of these did not pay for the transportation for these visits (median cost was \$0) and the median outof-pocket expenses for all these visits were AUD 40.

A total of $48(48 \%)$ participants underwent surgery, of whom 20 had CXL (including 3 patients who had 2 CXL procedures either on the same eye or on both eyes), 24 had a corneal transplant (including 3 who underwent multiple operations and 1 with a second/repeat corneal transplant) and 3 patients underwent both CXL and corneal surgery. The majority of CXL procedures were performed in a private facility $(n=14$, $58 \%$ ), while the majority of other surgeries were performed in a public hospital ( $n=18$, $64 \%$ ). Median expenditure for a single corneal transplant in a public hospital was AUD 200 (IQR 150, 300), in contrast to AUD 8975 (IQR 5500, 10000) when surgery was performed in a private hospital. A single CXL procedure performed in a public hospital 
cost AUD 25 (IQR 15, 50) while CXL in a private facility cost AUD 2995 (IQR 2000, 3225). Participants who had procedures in a private facility faced costs 6-45 times, on average, more than participants who had procedures performed in a public hospital. It is to be noted here that these data were collected prior to CXL being included in the Australia's Medicare Benefits Schedule and the estimated lifetime costs of the crosslinked patients may currently be slightly different.

A median of AUD 58 (IQR 0, 150) was spent by participants on eye drops. The median cost associated with the informal care and support from family members/carers was AUD 0 (IQR 0, 300) as the majority of the participants reported informed care being received by family members in-kind help (example: driving to and from the hospital).

In the preceding 12 months, participants estimated a median financial loss of AUD 500 (IQR 120, 1500) for their time away from employment to attend appointments, and other related inconveniences.

Table 3 represents the combined costs of $K C$ management. The median total cost of $K C$ was AUD 3365. The median expenditure for surgery was AUD 0 as it included those who underwent the surgery in a public hospital and had no related surgery costs (where expenses were recorded as AUD 0).

Table 3: Median expenses for activities for all participants involved. Costs reported are in Australian dollars. IQR, interquartile range.

Median AUD (IQR)

Medical equipment (spectacles, contact lenses $1088(650,1885)$ related, eye drops, other equipment)

Visits (optometrist, ophthalmologist, hospital, $378(100,1779)$ other health services)

Transportation to visits

$125(45,300)$ 


\begin{tabular}{ll}
\hline $\begin{array}{l}\text { Surgery } \\
\text { Direct costs (all of the above plus private health }\end{array}$ & $0(0,1150)$ \\
insurance) & \\
Total costs (direct plus informal care costs) & $3365(2110,895,6708)$ \\
\hline
\end{tabular}

Based on previous studies, ${ }^{17-20}$ Clinical KC eyes were classified into 3 subgroups according to front corneal curvature (Front $\mathrm{K}$ ) Front $\mathrm{K}$ readings (mild: Front $\mathrm{K} \leq 47.0$ Diopters (D), moderate: $47.0<$ Front $K<52.0$ D, and severe: Front $K \geq 52.0$ D). Using the worst eye for the overall severity there were 5 subjects with mild $\mathrm{KC}(5 \%)$, 25 with moderate KC (25\%), 29 with severe KC (29\%) and corneal curvature data was not available on the remaining 41 subjects. Kruskal wallis test was used for overall comparison costs related to $\mathrm{KC}$ between severities of $\mathrm{KC}$. There was no significant difference in total costs incurred by different subjects with varying degrees of $\mathrm{KC}$ (Table 4). However future studies are needed to confirm these results as we did not have the clinical data on all the subjects and also had a small group of subjects in the mild category of $\mathrm{KC}$.

Table 4: Comparison of costs incurred by subjects with varying degrees of keratoconus. Costs reported are in Australian dollars. IQR, interquartile range.

\begin{tabular}{|c|c|c|c|c|}
\hline \multirow[b]{3}{*}{$\begin{array}{l}\text { Total cost of } \\
\text { medical equipment } \\
\text { (spectacles, } \\
\text { contact lenses } \\
\text { related, eye drops, } \\
\text { other equipment) }\end{array}$} & \multicolumn{4}{|c|}{ Median AUD (I QR) } \\
\hline & Mild & Moderate & Severe & $\begin{array}{l}p- \\
\text { value }\end{array}$ \\
\hline & $\begin{array}{l}1600 \\
1920)\end{array}$ & $\begin{array}{l}1000 \\
1490)\end{array}$ & $\begin{array}{l}1410 \\
2150)\end{array}$ & 0.42 \\
\hline $\begin{array}{l}\text { Total cost related } \\
\text { to surgery }\end{array}$ & $0(0,0)$ & $60(0,2600)$ & $250(0,3000)$ & 0.19 \\
\hline $\begin{array}{l}\text { Total direct costs } \\
\text { (management, }\end{array}$ & $\begin{array}{l}2490 \\
2680)\end{array}$ & $\begin{array}{l}3030 \\
6575)\end{array}$ & $\begin{array}{l}3545 \\
9164)\end{array}$ & 0.28 \\
\hline
\end{tabular}




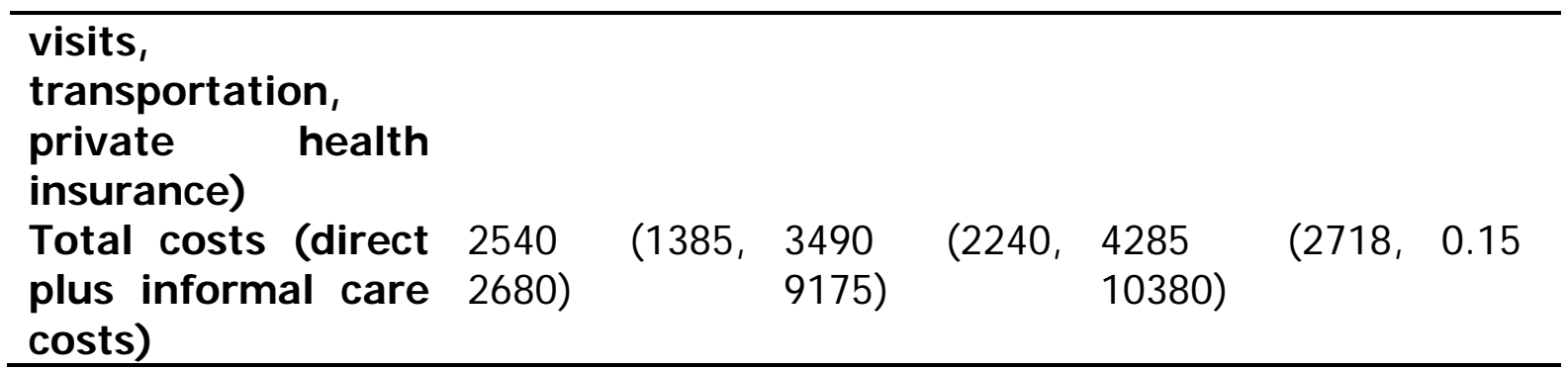

The estimated lifetime per capita cost ranged from AUD 3400 for those with a $<1$ year duration of KC to AUD 85,000 for those with 25 year's duration. The total yearly expenditure appeared greater in early years following KC diagnosis (Figure 1).

By applying our cost data to $K C$ prevalence data ( 1 in 2000) ${ }^{21}$, the total cost for $K C$ is estimated to be approximately AUD 44.7 million per year in Australia. The true cost may be higher if more recently estimated prevalence data in Denmark are used22.

Figure 1: Participant data by duration of disease

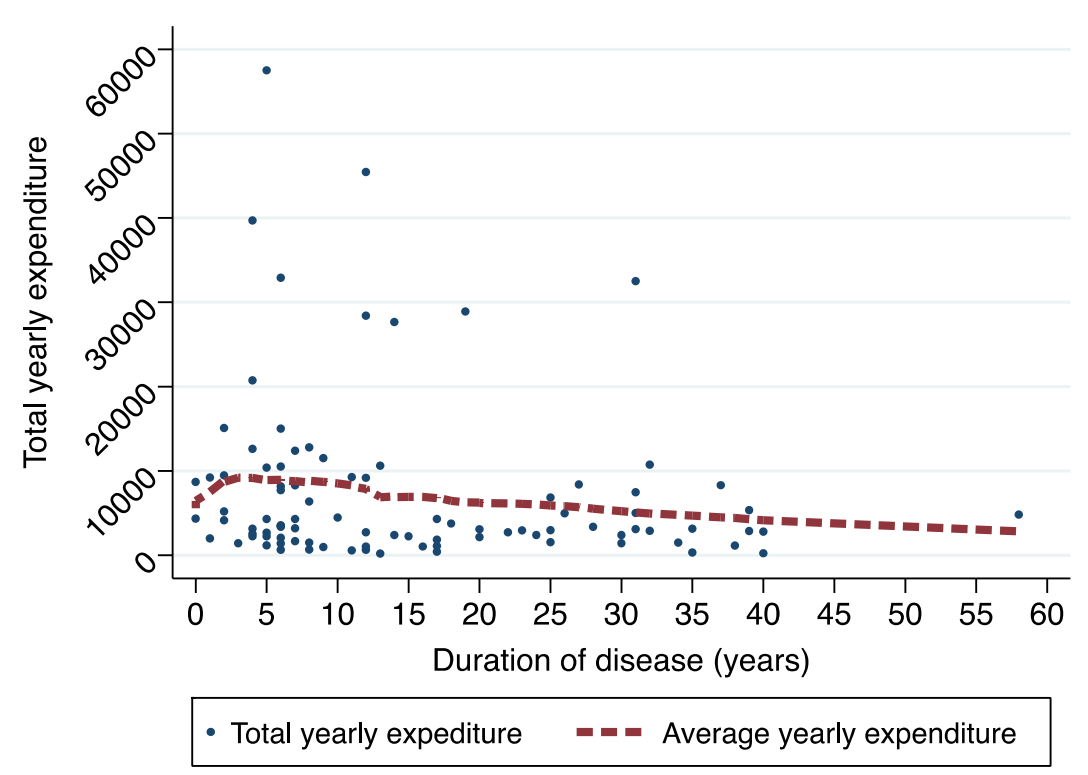




\section{DISCUSSION}

Our study reports the economic costs of KC in an Australian population where the patient bears "out of pocket" expenses for the costs related to the treatment and management of the condition. Our calculated median out-of-pocket cost was AUD 3365 over 12 months, which included costs for medical care, visual aids, private health insurance, productivity losses, transportation and indirect costs. The median total cost of KC was AUD 3400 while the average household optical expenditure was \$145 per annum (Australian households report 2008) ${ }^{16}$. This suggests KC patients are paying 30-fold more than the general population on the care of their eyes. Thus, the financial impact on the individual is important to consider in understanding the impact of this diagnosis.

Individuals with $\mathrm{KC}$ have a higher utilisation of eye care services than the general population. We found that $83 \%$ of participants had attended an optometrist, with $50 \%$ attending an optometrist at least 3 times in the preceding year. According to Australia's Medicare billings for optometric services, this is a much higher rate compared to the general population, where the average number of visits per person per year is 0.34 23 . This may be due to the finding that the majority $(61 \%)$ of participants wore contact lenses, which require more frequent visits for contact lens fitting and follow-up appointments. In the CLEK (Collaborative Longitudinal Evaluation of Keratoconus) study, $35 \%$ of individuals were refitted for their contact lenses in the year prior to their baseline visit ${ }^{24}$. Almost half of our cohort also saw an ophthalmologist (45\%) or attended a hospital clinic (52\%).

In Australia, 57\% of all people aged 18 years and over have private health insurance, of which $12 \%$ of individuals do not have ancillary cover (including optical expenses) ${ }^{25}$. This was approximately the same proportion in our cohort, where $55 \%$ had private 
insurance. Irrespective of cover, current government rebate and private health insurance policies do not fully cover all health-related expenses (including contact lens solutions, pharmaceuticals and visits to private ophthalmologists). Visual aids such as glasses and contact lenses are only partially covered by limited State-based subsidy schemes or by private health insurers (where a person's insurance includes ancillary optical cover). Thus, high out-of-pocket costs are faced by patients irrespective of their insurance status. In fact, in our study, participants who had procedures in a private facility faced costs 6 to 45 times more than those who had procedures performed in a public hospital. This may partly explain the high (78\%) level of dissatisfaction amongst participants regarding their private health cover.

In the CLEK study, $11.5 \%$ of their cohort reported missing work due to $\mathrm{KC}^{24}$, highlighting the impact that the condition may have on income and productivity. The high cost is particularly significant as only $58 \%$ of our participants were employed at the time of completion of the questionnaire.

In contrast to our study, Rebenitsch et al estimated the lifetime economic burden of KC in 2007 from a health care service perspective using a simulated cohort based on published sources of information, physicians' consultations and authors' own estimations. They calculated an average annual cost of USD 653 for treating $\mathrm{KC}$ and a lifetime cost of USD 24168 above routine eye care for myopia. This contrasted to our estimated median cost of AUD 3365 (equivalent to USD 2312) per year. Some of the reasons for this discrepancy can be due to different health systems and health care models, a more complete recording of expenditures on our study, and the availability of more treatments and increases in costs over the past decade. The true economic burden of $K C$ to the community and the health system includes both the cost to the health system and to the individual. 
Rebenitsch et al. estimate might be the actual life-time costs (which includes many years where there is no disease progression), while our sample might be an underestimation as we are more likely to pick only patients that are in the progressive stage when they come to clinic. Our study and Rebenitsch's study have completely different design (cross-sectional self-estimation of the costs vs a simulation study based on estimates from CLEK patients) and therefore any comparisons are purely descriptive.

Any long-term modelling of the economic impact of $\mathrm{KC}$ on the community would also have to include the effect of CXL. As CXL has been shown to halt the progression of the disease in most treated patients, this would be expected to reduce the long-term costs of the disease, with a reduction in the need for changes in spectacle or contact lens prescriptions, visits to optometrists and ophthalmologists, rates of corneal transplantation ${ }^{26}$ and loss of productivity for patients and their family/carers while attending appointments. This was not accounted for in Rebenitsch et al's and our study in estimating the lifetime costs of KC. Rebenitsch, et al did not include CXL in the analysis as it was not FDA approved at the time when they conducted the economic burden study. However, in Melbourne, CXL has been available since 2006 and therefore its influence on costs incurred by our participants should already be felt.

The main limitation of our study is its reliance on requiring participants to estimate their costs retrospectively. In addition, it only addresses costs over a 12-month period. To minimise selection bias in our cohort, patients from both private and public hospital clinics were approached for inclusion in the study. A prospective study could confirm the results in this study.

The results of this study highlight the economic impact of $\mathrm{KC}$ on individuals. $\mathrm{KC}$ usually has its onset during the teenage years, progressing throughout an individual's core 
education, prime earning and child-rearing years. We have previously reported that the vision-related quality of life utility value is lower in individuals with $\mathrm{KC}$ compared to those with diabetic retinopathy or age-related macular degeneration. ${ }^{12}$ With both impaired vision-related quality of life and a significant economic impact, $\mathrm{KC}$ represents a public health concern.

\section{Acknowledgements}

The authors would like to thank Keratoconus Australia, A/Prof Grant Snibson, Mr Richard Lindsay and REDCap.

\section{REFERENCES}

1. Rabinowitz YS. Keratoconus. Surv Ophthalmol 1998; 42(4):297-319.

2. Wittig-Silva C, Chan E, Islam FM, et al. A randomized, controlled trial of corneal collagen cross-linking in progressive keratoconus: three-year results. Ophthalmology 2014;121(4):812-21. 
3. Wittig-Silva $\mathrm{C}$, Whiting $\mathrm{M}$, Lamoureux $\mathrm{E}$, et al. A randomized controlled trial of corneal collagen cross-linking in progressive keratoconus: preliminary results. J Refract Surg 2008;24(7):S720-5.

4. Hashemi H, Seyedian MA, Miraftab M, et al. Corneal collagen cross-linking with riboflavin and ultraviolet a irradiation for keratoconus: long-term results. Ophthalmology 2013;120(8):1515-20.

5. McAnena L, Doyle F, O'Keefe M. Cross-linking in children with keratoconus: a systematic review and meta-analysis. Acta Ophthalmol 2016.

6. Chang CY, Hersh PS. Corneal collagen cross-linking: a review of 1-year outcomes. Eye Contact Lens 2014;40(6):345-52.

7. Agrawal V. Long-term results of cornea collagen cross-linking with riboflavin for keratoconus. Indian J Ophthalmol 2013;61(8):433-4.

8. KA Williams, MC Keane, RA Galettis, et al. The Australian Corneal Graft Registry 2015 Report. 2015.

9. The Australian Corneal Graft Registry. 1990 to 1992 report. Aust N Z J Ophthalmol 1993;21(2 Suppl): 1-48.

10. Rebenitsch RL, Kymes SM, Walline JJ, Gordon MO. The lifetime economic burden of keratoconus: a decision analysis using a markov model. Am J Ophthalmol 2011;151(5): 768-73 e2.

11. Sahebjada S, Schache M, Richardson Al, et al. Association of the hepatocyte growth factor gene with keratoconus in an Australian population. PLoS One 2014;9(1):e84067.

12. Sahebjada S, Fenwick EK, Xie J, et al. Impact of keratoconus in the better eye and the worse eye on vision-related quality of life. Invest Ophthalmol Vis Sci 2014;55(1):412-6.

13. Sahebjada S, Schache M, Richardson AJ, et al. Evaluating the association between keratoconus and the corneal thickness genes in an independent Australian population. Invest Ophthalmol Vis Sci 2013;54(13):8224-8. 
14. Harris PA, Taylor $\mathrm{R}$, Thielke $\mathrm{R}$, et al. Research electronic data capture (REDCap)--a metadata-driven methodology and workflow process for providing translational research informatics support. J Biomed Inform 2009;42(2):377-81.

15. Kennedy RH, Bourne WM, Dyer JA. A 48-year clinical and epidemiologic study of keratoconus. Am J Ophthalmol 1986;101(3):267-73.

16. http://www.abs.gov.au/ausstats/abs@.nsf/mf/3101.0.

17. Abolbashari F, Mohidin N, Ahmadi Hosseini SM, et al. Anterior segment characteristics of keratoconus eyes in a sample of Asian population. Cont Lens Anterior Eye 2013;36(4):191-5.

18. Kymes SM, Walline JJ, Zadnik K, et al. Quality of life in keratoconus. Am J Ophthalmol 2004; 138(4):527-35.

19. Chopra I, Jain AK. Between eye asymmetry in keratoconus in an Indian population. Clin Exp Optom 2005;88(3):146-52.

20. Sahebjada S, Xie J, Chan E, et al. Assessment of anterior segment parameters of keratoconus eyes in an Australian population. Optom Vis Sci 2014;91(7):803-9.

21. Jonas J B, Nangia V, Matin A, et al. Prevalence and associations of keratoconus in rural maharashtra in central India: the central India eye and medical study. Am J Ophthalmol 2009; 148(5): 760-5.

22. Godefrooij DA, de Wit GA, Uiterwaal CS, et al. Age-specific Incidence and Prevalence of Keratoconus: A Nationwide Registration Study. Am J Ophthalmol 2017;175:169-72.

23. Gerrits W, Labussiere E, Dijkstra J, et al. Letter to the Editor: Recovery test results as a prerequisite for publication of gaseous exchange measurements. J Anim Sci 2017;95(12):5175.

24. Wagner H, Barr JT, Zadnik K. Collaborative Longitudinal Evaluation of Keratoconus (CLEK) Study: methods and findings to date. Cont Lens Anterior Eye 2007;30(4):223-32. 
25. Metge F, Czaja-Hasse LF, Reinhardt R, Dieterich C. FUCHS-towards full circular RNA characterization using RNAseq. PeerJ 2017;5: e2934.

26. Godefrooij DA, Gans R, Imhof SM, Wisse RP. Trends in penetrating and anterior lamellar corneal grafting techniques for keratoconus: a national registry study. Acta Ophthalmol 2016; 94(5):489-93. 


\section{Economic Burden Questionnaire}

Please fill in the questionnaire for everything which is "related to keratoconus only".

Please DO NOT include:

- Glasses/contact lenses/solutions/medicines/services you buy for someone else

- Medicines and health care services you seek which are not related to keratoconus

Thank you!

Please fill in the questionnaire for everything which is "RELATED TO KERATOCONUS ONLY".

Please DO NOT include:

- Glasses/contact lenses/solutions/medicines/services you buy for someone else

- Medicines and health care services you seek which are not related to keratoconus

How often do you wear glasses to see objects clearly?

Daily

Several times per week

On occasion

$\bigcirc$ Never

How many pairs of glasses did you purchase in the last 12 months?

When did you last purchase a pair of glasses to see objects clearly?

Less than 6 months ago

$6-12$ months ago

12 - 24 months ago

More than 24 months ago

What was the total out of pocket payment (that was not claimable through private health insurance) for your glasses in the last 12 months? (in AUD)

How often do you wear contact lenses to see objects clearly?

Daily

Several times per week

On occasion

Never

What was the total out of pocket payment (that was

NOT claimable through Medicare or private health

insurance) for the contact lenses in the last 12

months? (in AUD)

How much do you spend on the care of your contact

lenses in a year? (e.g. Solutions, cases,

lubricating drops etc.) (in AUD) 


\section{COSTS RELATED TO PRIVATE HEALTH INSURANCE}

Do you have a private health insurance that has an $\bigcirc$ Yes

extras cover for optical benefits?

No

How much do you spend on private insurance per year?

(in AUD)

Are you satisfied with the rebates provided to you

for contact lenses and glasses by the insurers?

Y Yes

No

Please explain

$\begin{array}{ll}\text { Would you be willing to pay higher premiums in order } & \bigcirc \text { Yes } \\ \text { to receive higher rebates in relation to the cost of } & \text { No }\end{array}$

contact lenses and glasses (i.e. optical cover)?

The reason for not having a private health insurance

is because-

$\square$ No need

$\square$ Too expensive

$\square$ Poor value for money

$\square$ Others

Please specify 'other' reasons

What other expenses should be covered in the rebates

for optical cover?

Contact lens solution

Contact lens fitting fees

$\square$ Surgical fees

$\square$ Eye drops

$\square$ Others

Please specify 'other' expenses

\section{COSTS RELATED TO KERATOCONUS MANAGEMENT}

16 Please fill in the following section, relating to the appropriate services that you received in the past 1 year for keratoconus.

Please check if the bill or receipt is available for each visit.

Did you at any time during the past 1 year receive care from an OPTOMETRIST for your keratoconus?

$\bigcirc$ Yes

No

Please specify number of visits to the Optometrist

What type of clinic/s did you go to?

$\square$ Private

$\square$ Public

Both 
What was the purpose of these visits?
Contact lens fitting
$\square$ Contact lens complication
$\square$ Follow up
Routine Check up
$\square$ Referral
$\square$ Prescription check for glasses
$\square$ Others

Please specify 'other' purposes

What was the total cost of transportation for all these visits? (in AUD)

In the out of pocket expenses, please include- examination fees, medication, contact lens fitting fees, imaging fees, hospital admission fees, surgeon's fee, anaesthetist fees.

DO NOT INCLUDE costs of glasses, contact lenses, contact lens solutions.

What was the total out of pocket expenses for all these visits? (in AUD)

Did you at any time during the past 1 year receive care from an OPHTHALMOLOGIST for your keratoconus?

$\bigcirc$ Yes

No

Please specify number of visits to the

Ophthalmologist

What type of clinic/s did you go to?
$\square$ Public
$\square$ Private
$\square$ Both

\section{What was the purpose of these visits?}

$\square$ Contact lens fitting
$\square$ Contact lens complication
$\square$ Follow up
$\square$ Routine Check up
$\square$ Referral
$\square$ Prescription check for glasses
$\square$ Surgery
$\square$ Others

Please specify 'other' purposes

What was the surgery that you underwent?(E.g.:

Corneal Transplant; Cross linking etc.)

What was the total cost of transportation for all these visits? (in AUD)

In the out of pocket expenses, please include- examination fees, medication, contact lens fitting fees, imaging fees, hospital admission fees, surgeon's fee, anaesthetist fees.

DO NOT INCLUDE costs of glasses, contact lenses, contact lens solutions. 
What was the total out of pocket expenses for all these visits? (in AUD)

Did you at any time during the past 1 year receive care from an EYE HOSPITAL/CLINIC for your

Yes

keratoconus?

No

(Please do not include the above mentioned

Optometrist/Ophthalmologist visits)

Please specify number of visits to the Eye Hospital

What type of clinic/s did you go to?

$\square$ Public

Private

Other

What was the purpose of these visits?
Contact lens fitting
Contact lens complication
Follow up
Routine Check up
Referral
Prescription check for glasses
$\square$ Surgery
Others

Please specify 'other' purposes

What was the surgery that you underwent?(E.g.:

Corneal Transplant; Cross linking etc.)

What was the total cost of transportation for all these visits? (in AUD)

In the out of pocket expenses, please include- examination fees, medication, contact lens fitting fees, imaging fees, hospital admission fees, surgeon's fee, anaesthetist fees.

DO NOT INCLUDE costs of glasses, contact lenses, contact lens solutions.

What was the total out of pocket expenses for all these visits? (in AUD)

Did you at any time during the past 1 year receive care from any of other health services for your $\bigcirc$ Yes keratoconus?

No

Which of these health services have you been to?
$\bigcirc \mathrm{GP}$
Psychologist
Psychiatrist
Homeopath
$\bigcirc$ Trad. Chinese Medicine
Masseur
Social support services
Personal care services
$\bigcirc$ Others 
Please specify 'others'

What was the total cost of transportation for all

these visits? (in AUD)

What was the total out of pocket expenses for all

these visits? (in AUD)

\section{COSTS RELATED TO INFORMAL CARE \& SUPPORT}

What is your employment status?

$\bigcirc$ Employed for wages

$\bigcirc$ Self-employed

A homemaker

A student

$\bigcirc$ Retired

$\bigcirc$ Unable to work because of keratoconus

Did you have to change your subjects/course due to your eye condition?

Yes
No

Please explain

What is your total household income?
Less than $\$ 10,000$
$\$ 10,000$ to $\$ 19,999$
$\$ 20,000$ to $\$ 29,999$
$\$ 30,000$ to $\$ 39,999$
$\$ 40,000$ to $\$ 49,999$
$\$ 50,000$ to $\$ 59,999$
$\$ 60,000$ to $\$ 69,999$
$\$ 70,000$ to $\$ 79,999$
$\$ 80,000$ to $\$ 89,999$
$\$ 90,000$ to $\$ 99,999$
$\$ 100,000$ to $\$ 149,999$
$\$ 150,000$ or more
$\bigcirc$ Prefer not to mention

In the past 1 year, approximately what monetary value would you assign for the time taken off work to attend clinics and also other inconveniences caused due to keratoconus? (in AUD)

Please read the list of examples below to explain the type of information you need to fill in the next section.

\section{Example A Type of Care}

- Home help such as household tasks, preparing meals, gardening, household repairs

- Personal affairs such as paying bills, writing letters, banking, shopping, buying groceries, organising services such as meals from food preparation organisation or home help, arranging appointments.

- Communication such as reading mail or books for you, sitting in on appointments with you.

- Transport such as driving to and from appointments and the shops

Example B Who provided the care/support

- Wife, husband or partner 


\section{Mother or father}

- Son or daughter

- Other relative - please write in their relationship to you: eg : Granddaughter

- Friend or neighbour

\section{Example C Cost to you or the helper}

Please write down the amount of cost, who paid the amount and what was this payment for.

\section{Examples:}

- \$3.00 (son - car parking)

- \$15.00 (self - home help)

- \$0 (daughter - writing letters)

Please specify the type of care (example A),

provider/helper (example B) and the cost to you or

the helper (example $\mathrm{C}$ ).

E.g.: Transport - Husband - \$30 (self-petrol)

In the past 1 year, have you used any of the

following medicines, products and equipment for

keratoconus?
Prescription medicines, tablets, eye drops, etc. Non-prescription medicines, tablets, eye drops, etc.

Products: e.g. Low vision device, magnifier, cane, etc.

Equipment: e.g. Special television or computer screen, special computer software and telephone modifications

Other items

Please specify 'other items'

Please list the items purchased in the last 1 year, along with the total out of pocket expenses for each of the listed items.

E.g.: 1) Eye drops - $\$ 60$

2) Special Computer screen - $\$ 300$ 


\section{University Library}

\section{- M M N E R VA A gateway to Melbourne's research publications}

Minerva Access is the Institutional Repository of The University of Melbourne

Author/s:

Chan, E;Baird, PN;Vogrin, S;Sundararajan, V;Daniell, MD;Sahebjada, S

Title:

Economic impact of keratoconus using a health expenditure questionnaire: A patient perspective

Date:

2020-01-09

Citation:

Chan, E., Baird, P. N., Vogrin, S., Sundararajan, V., Daniell, M. D. \& Sahebjada, S. (2020). Economic impact of keratoconus using a health expenditure questionnaire: A patient perspective. CLINICAL AND EXPERIMENTAL OPHTHALMOLOGY, 48 (3), pp.287-300. https://doi.org/10.1111/ceo.13704.

Persistent Link:

http://hdl.handle.net/11343/275254 